\title{
Rigid and Deformable Vasculature-to-Image Registration: A Hierarchical Approach
}

\author{
Julien Jomier and Stephen R. Aylward \\ Computer-Aided Diagnosis and Display Lab \\ The University of North Carolina at Chapel Hill, Department of Radiology \\ 27510 Chapel Hill, USA \\ $\{$ jomier, aylward\}@unc.edu
}

\begin{abstract}
Several recent studies demonstrate the potential of using tubular structures such as vessels as a basis for image registration. In this paper, we present a novel technique for the deformable registration of tubular structures. Our approach aligns tubular models, e.g. vessels of an organ, with an image by combining both rigid and non-rigid transformations in a hierarchical manner. The physical structure and properties of the vessels are taken into account to drive the registration process. Our model-to-image registration method shows sub-voxel accuracy as well as robustness to noise and a convergence time of less than one minute.
\end{abstract}

\section{Introduction}

Registration of vascular images has shown promising results compared to tissue based registration [3] and has been used to form vascular atlases [4 in order to diagnose cerebral malformations. Other extensions to this technique have also been applied to image-guided surgery.

Registration methods typically use mutual information 911] and related image-image metrics to register one image with another. Iterative closest point is also used to perform model-model registration of common features extracted from two images. A third type of registration is used in [1: features from one image are registered directly with another image using a model-image match metric. However the extension of this method to deformable registration has not been shown.

A variety of deformation field estimation methods exist. Fluid based, imageimage registration approaches [7] handle arbitrary deformations but do not take advantage of the object's geometry in images. Finite element modeling also shows excellent results [6] by deforming a mesh given image forces. On the other hand, model-to-model registration techniques may directly exploit the geometric correspondences have been developed. Our technique differs from these approaches by (1) combining both rigid and deformable transformations in a hierarchical manner, (2) combining geometry and intensity information and (3) persisting as an instance of model-to-image registration.

Our technique takes advantage of the typical tree structure of blood vessels and uses branch points to constrain the deformation field. We perform three 
distinct steps to achieve final registration of the model with the image: global rigid transformation, piece-wise rigid registration and deformable registration. The first stage deals with the global rigid body registration and has been shown to have sub-voxel accuracy, handle large initial mis-registrations and converge in 2-10 seconds 1]. Such rigid registration is a preliminary and necessary stage in order to be "close enough" to the deformed structure. The second stage uses the tree structure inherent in vascular network to perform a piece-wise rigid alignment. First, the root of the tree is aligned and then its children are registered, in order, from root to leaves. Branch points and physical parameters of the tubular structure have to be known to approximately constrain this task. Hierarchical local deformation is the concern of the third stage.

\section{Method}

Blood vessels in the human body are organized as a tree structure. For instance, in the liver, portal and hepatic vessels define two distinct trees; in the brain, vessels are divided into several trees, among them, right and left cerebral group. Formally, a tree is composed of at least one root, but vasculature trees can have multiple roots and can contain cycles. Our technique relies on this tree configuration to perform a global to local registration. First, a 3-dimensional model of the vasculature is formed using a ridge traversal technique [2]. Each extracted blood vessel is represented as a centerline with an associated radius at each point on the line. Next, we initiate our deformable registration strategy by solving for a global rigid transform.

\subsection{Global Rigid Registration}

Our rigid registration method maps a 3-dimensional vascular model into the target image using a tube-to-image technique developed by Aylward et al. [1]. This algorithm relies on blood vessels to have high intensity values in the target image. For each sample point of the model, the intensity is computed in the target image at a scale proportional to the radius of the vessel at that point. The sum of these intensities is the value of the match metric and the parameters of the transformation are optimized to maximize this metric. A unique additional property of this method is that it limits vessel to inducing registration updates in their normal directions. Furthermore, the iterative updates of the rigid transform are adjusted for the orientation bias of the vessels. The second step consists of a piece-wise rigid registration from root to leaves.

\subsection{Piece-Wise Rigid Transformation via Propagation}

A rigid registration is applied to each vessel in a hierarchical manner. As in our global rigid registration step, we align the model to match high intensity values in the target image. First the root of the tree is registered with the image using a rigid body transformation. Second, the branches of the tree are registered rigidly 
with the image one branch at a time using the parent-child hierarchy with anchor points at the branch points. That is during this step we solve for the rotation at each branch point using the parent-child hierarchy.

The magnitude of the rotation is given by the displacement vector $\boldsymbol{v}$ computed along each branch individually (sub-branches do not contribute). The evaluation of the rotation is done using a linear weighted factor $\lambda(i)$ along the child tube so that points close to the branch contribute more to the rotation. The image gradient is computed only at centerline points $x$ at a scale $\sigma$ proportional to the radius of the tube at that point. $N$ represents the number of centerline points that compose the vessel. For each centerline point $i$ the image gradient is projected onto its normal plane $\boldsymbol{n}_{\boldsymbol{i}}=\left(\boldsymbol{n}_{\mathbf{1}}, \boldsymbol{n}_{\mathbf{2}}\right)_{i}$.

$$
\boldsymbol{v}=\frac{1}{N} \sum_{i=1}^{N} \lambda(i) \boldsymbol{\nabla}_{\boldsymbol{x}}(\sigma) \cdot \boldsymbol{n}_{\boldsymbol{i}}
$$

To translate a branch, the elastic property of the parent has to be taken into account. Specifically, the translation vector $\boldsymbol{v}$ of the child is projected onto the tangent direction $\boldsymbol{t}$ of its parent at the specified branch point $x$ and the amount of translation $\boldsymbol{T}$ allowed is constrained by the elasticity $\gamma$ of the parent tube multiplied by the initial distance $d$ between the two consecutive points near the branch.

$$
\boldsymbol{T}=\max \left(\boldsymbol{v} \cdot \boldsymbol{t}, \gamma d-\left|x-x_{0}\right|\right) \cdot \boldsymbol{t}
$$

Once the branch point is moved, the points of the parent are updated to propagate the translation along the tube. We repeat the process until convergence before going to the next step: the deformable registration.

\subsection{Deformable Registration}

Non-rigid registration is also driven by derivative information from the target image. Our approach uses the image gradient computed at a scale proportional to the radius of the tube and projected onto the normal of the tube. Due to the potential complexity of the elastic deformations, i.e. folding, shrinking, expansion, etc., we add constraints to the registration process.

The first constraint is the elasticity coefficient $\gamma$ of the tube which limits the movement of points along a tube. This is the same as 2.1 but now everypoint along a tube may move.

The second constraint is the rigidity coefficient which defines the bending factor of the tube. There are several ways to define such a coefficient. We define rigidity as the maximum angle between the initial tangent $\boldsymbol{t}_{\mathbf{0}}$ at rest and the actual tangent $\boldsymbol{t}$. The rigidity coefficient can be different for each point along the structure or can be constant. Intuitively, the rigidity of a vessel is proportional to its radius and depends on its material and physical properties. In our implementation we choose to keep the rigidity constant and use a non-uniform sampling rate to accommodate the rigidity coefficient as the radius changes. 
An iterative optimization process uses these two coefficients and the projected gradient at each point along a centerline to fit each centerline to the data. This continues until the full hierarchy has been fit to the data.

\section{Results}

In order to evaluate the accuracy of our registration algorithm we compared registered blood vessels with vessels that had been extracted from the target image. It is important to note that the extracted target image vessels are not used in the registration process, but only for validation purposes.

\subsection{Simulated Data}

First, we tested our algorithm on simulated data to perform noise sensitivity measurements. We created an artificial tree composed of a main trunk and two branches. The main trunk is a straight tube composed of 50 points while the branches are 20 points long. The three tubes have a constant radius of $2 \mathrm{~mm}$. Next, we deformed the tree and created a binary image of the deformed tree such that high intensity pixels fall inside the tubes. Finally, the image is blurred by a Gaussian filter $(\sigma=5)$ since blood vessel's cross section have a Gaussian profile in clinical data. Figure 1 shows a slice of the synthetic image.
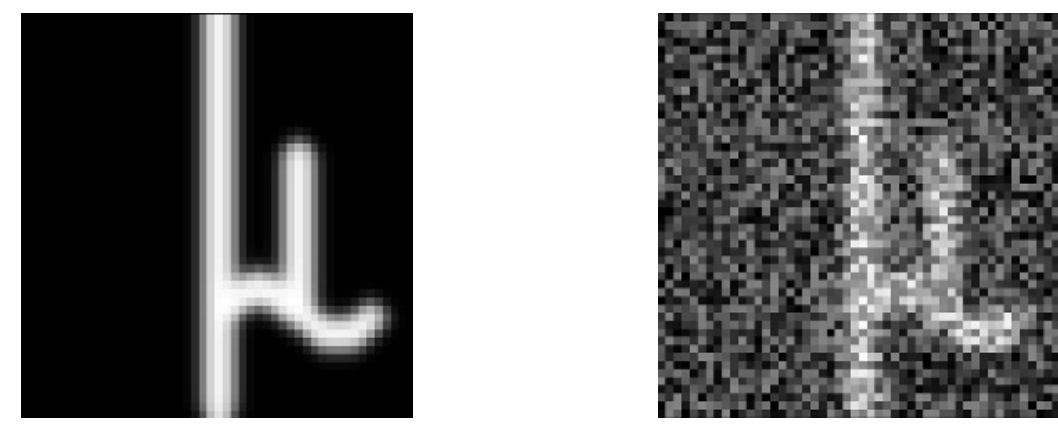

Fig. 1. Simulated image, with no noise(left) and with a noise range [0,255](right), used to test the robustness to noise of our algorithm.

Figure 2] shows the three consecutive steps of the algorithm, after rigid registration(left), after piece-wise rigid transformations(middle) and after non-rigid registration(left). Before and after the registration process we compute the cumulated measures of the percentile of points inside a given distance from the centerline using the closest point metric. Results are shown in Figure 3.

Next, we quantify the robustness to noise of our algorithm by adding uniform additive noise to the simulated image (Figure 1-right). Table 4 presents the results of the registration given different ranges of noise level. The accuracy of 


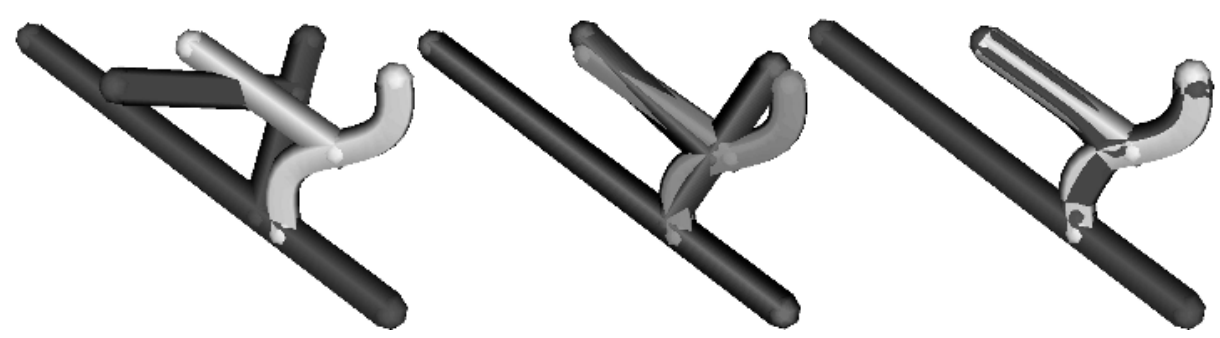

Fig. 2. Simulated tubes used to test the robustness of our algorithm. Original sets of tubes(left), After semi-rigid registration(middle) and after non-rigid registration(right). Only the light grey tubes are moving, they are being registered with the deformed image. The dark vessels are only being shown to illustrate "truth"
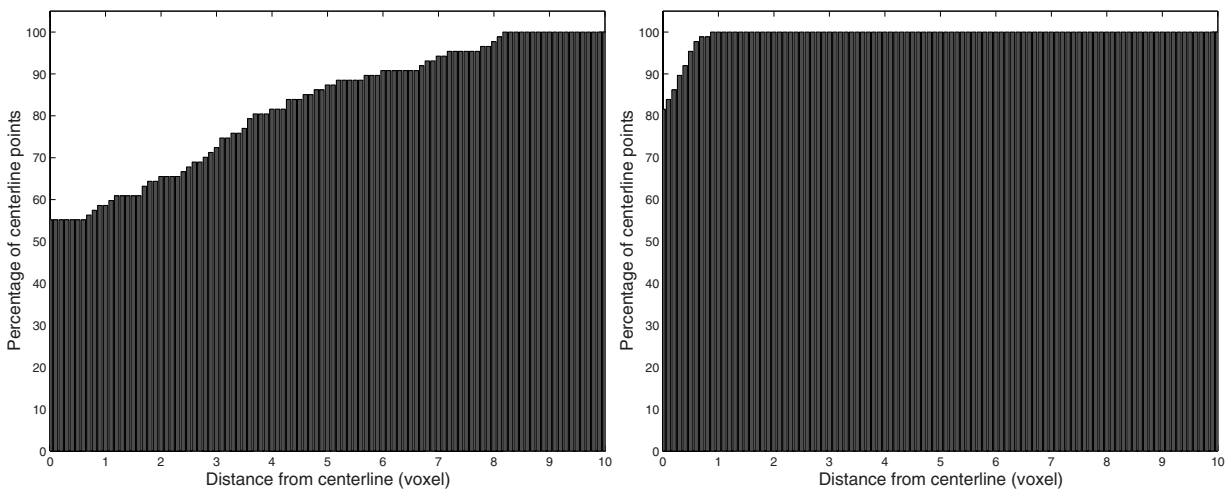

Fig. 3. Cumulative graphs representing the percentile of vascular points within a certain distance from the centerline of the true vasculature point, before registration(left) and after rigid and non-rigid transformations(right)

the registration process shows a fall-off of $3.5 \%$ only even when the noise pans the same range as the image. The summation of derivatives calculated using gaussians with standard deviation proportional to the size of the object is very robust to noise [8].

Noise level range $\%$ of points $\leq 1$ voxel $\%$ of points $\leq 2$ voxels $\mid \%$ of points $>2$ voxels

\begin{tabular}{c|c|c|c}
\hline$[0,0]$ & $100 \%$ & $100 \%$ & $0 \%$ \\
\hline$[0,50]$ & $100 \%$ & $100 \%$ & $0 \%$ \\
\hline$[0,100]$ & $97.7 \%$ & $100 \%$ & $0 \%$ \\
\hline$[0,200]$ & $96.8 \%$ & $99.7 \%$ & $0.3 \%$ \\
\hline$[0,255]$ & $96.5 \%$ & $99 \%$ & $1 \%$
\end{tabular}

Fig. 4. Influence of different additive white noise levels on the registration process 


\subsection{Pre-post-surgery Brain Data}

We applied our algorithm on pre- and post-surgery brain Time-of-flight MRA data in which an arteriovenous malformation (AVM) had been embolized. The data volume sizes $256 \times 256 \times 104$ and has been isotropically resampled to a spacing of 0.87 . Approximately 100 vessels were extracted with an average of 150 points per vessel. An initial global rigid registration was performed using a sample factor of 10, i.e. approximately 15 points per vessel are used for registration. Fig 5 left shows the result. Next we applied 40 piece-wise rigid transformation iterations per branch, Fig 5 right. Fig 6 shows the final registration using both piece-wise rigid and non-rigid transformations.
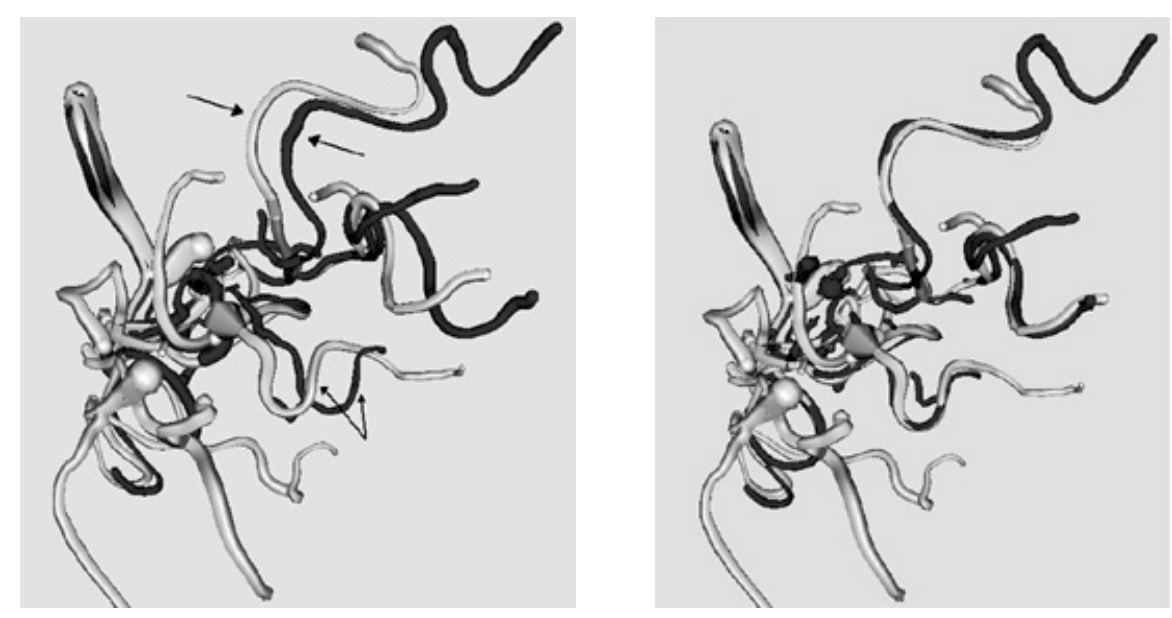

Fig. 5. Original set of tubes registered with a global rigid transformation(left) and the resulting set of tubes after the piece-wise rigid registration(right). Only the black vasculature is moving. The light grey vasculature is shown here as truth but is never used to drive the registration process. The data that produced the clinical MRA light grey tubes is actually driving the registration process

After each stage of the registration process we compute the percentile of centerline points inside a given distance from the centerlines of the vessels in the target image. Figure 7 shows the results. Both stages of the deformable registration requires less than 10 seconds to converge on a standard desktop PC Pentium $4(2.4 \mathrm{GHz})$ without any parallelization. Depending on the complexity of the vascular tree (number of branches, size of the tree) the computation time can be decreased significantly using parallel processing [1]. We are currently integrating our algorithm for Radio-Frequency Ablation (RFA) of liver tumors in the operating room and are pursuing parallelization and other speed improving enhancements. 


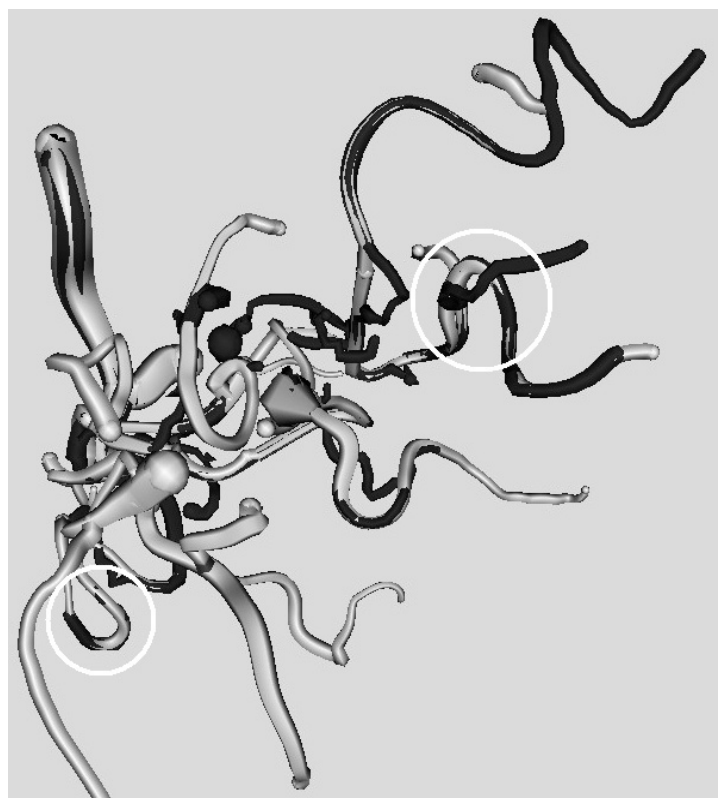

Fig. 6. Set of tubes after the deformable registration process. Non-rigid deformations are applied after the semi rigid registration. Again, the light grey vasculature is shown here as an illustration but is never used to drive the registration process. Circles highlight areas of larger non-rigid deformation
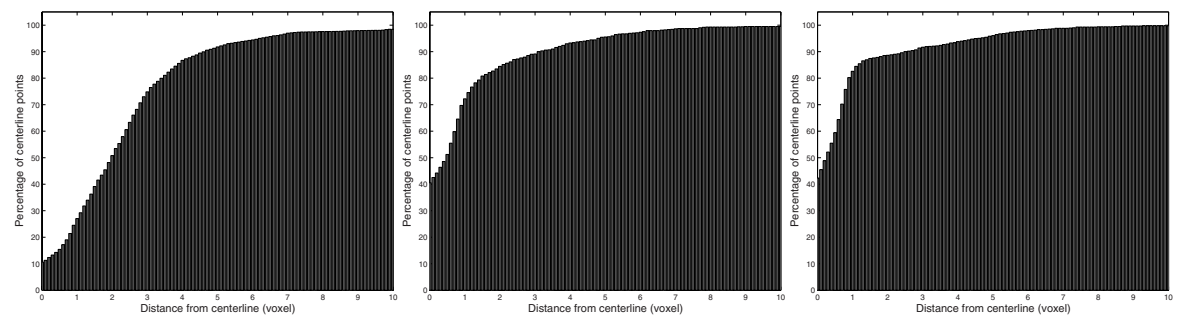

Fig. 7. Percentile of points inside a given distance from the centerlines of the vessels in the target image (cumulative graphs). Before registration(left), after semi-rigid registration(middle) and after semi-rigid plus deformable registration(right)

\section{Discussion and Conclusions}

We have developed a novel model to image registration technique that uses both rigid and deformable transformations in a hierarchical manner. The model, a set of blood vessels, is registered with the image by exploiting the parent-child hierarchy present in the vasculature. Furthermore, elasticity and rigidity coefficient of the vessels are taken into account during the registration process to constrain the deformation field. Our algorithm shows high accuracy and robustness to 
noise on simulated data and operate with $\approx 87 \%$ of centerline points within 2 voxels on pre-to-post AVM embolization MRA registration. We are conscious that our definitions of elasticity and rigidity do not have "real" physical meaning and we are currently investigating the physical utility of incorporating the properties of blood vessels embedded in different tissues.

Acknowledgements. This work is funded in part by the Whitaker Foundation (RG-01-0341), the NIH-NIBIB (R01 EB000219), and the NIH-HLB (R01 HL69808).

\section{References}

1. Aylward, S., Jomier, J., Weeks, S., Bullitt, E.: Registration of Vascular Images International Journal of Computer Vision, November 2003, pages 15

2. Aylward, S., Bullitt, E.: Initialization, Noise, Singularities, and Scale in HeightRidge Traversal for Tubular Object Centerline Extraction IEEE Transactions on Medical Imaging, Feb, 2002, Pages 61-75

3. Cool, D., Chillet, D., Kim, J., Foskey, M., Aylward, S.: Tissue-Based Affine Registration of Brain Images to form a Vascular Density Atlas MICCAI 2003, November 2003

4. Chillet, D., Jomier, J., Cool, D., Aylward, S.: Vascular Atlas Formation Using a Vessel-to-Image Affine Registration Method MICCAI 2003, November 2003

5. Danielsson, P.E.: Euclidean distance mapping Computer Graphics and Image Processing, 14, 1980, pp. 227-248.

6. Ferrant, M., Warfield, S., Guttmann, C., Mulkern, R., Jolesz, F., Kikinis, R.: 3D Image Matching Using a Finite Element Based Elastic Deformation Model MICCAI 1999,pp 202-209

7. Lorenzen, P. J., Joshi, S. C.: High-Dimensional Multi-modal Image Registration. WBIR 2003: 234-243

8. Lindeberg, T.: Linear Spatio-Temporal Scale-Space. Scale-Space 1997: 113-127

9. Maes, F., Collignon, A., Vandermeulen, D., Marchal, G., Suetens, P.: Multimodality image registration by maximization of mutual information IEEE Transactions on Medical Imaging, vol. 16, no. 2, pp. 187-198, April 1997.

10. Maintz, J.B.A., Viergever, M.A.: A Survey of medical image registration. In U. Spetzger, H.S. Stiehl, J.M. Gilsbach (Eds.), Navigated Brain Surgery (pp. 117136). Aachen: Verlag Mainz.

11. Rueckert, D., Clarkson, M. J., Hill,D. L. G., Hawkes,D. J.: Non-Rigid Registration Using Higher-Order Mutual Information. Proc. SPIE Medical Imaging 2000: Image Processing, pp. 438-447

12. Wink, O., Niessen, W.J., Verdonck, B., Viergever, M.A.: Vessel Axis Determination Using Wave Front Propagation Analysis MICCAI 2001,LNCS 2208, pp. 845-853, 2001 . 